\title{
Transforming the Shock Pattern of Supersonic Jets using Fluidic Injection
}

\author{
Bernhard Semlitsch* \\ Whittle Laboratory, Department of Engineering, University of Cambridge, 1 JJ Thomson Avenue, Cambridge, \\ CB3 0DY, United Kingdom \\ Daniel R. Cuppoletti ${ }^{\dagger}$ \\ National Research Council, Air Force Research Laboratory, Wright-Patterson AFB, OH 45433, USA \\ Ephraim J. Gutmark ${ }^{\ddagger}$ \\ Department of Aerospace Engineering, University of Cincinnati, 799 Rhodes Hall, Cincinnati, OH \\ 45221-0070, USA \\ Mihai Mihăescu ${ }^{\S}$ \\ Linné FLOW Centre, Department of Mechanics, Royal Institute of Technology (KTH), Osquars Backe 18, \\ 10044 Stockholm, Sweden
}

\begin{abstract}
Double shock diamonds establish in the exhaust of modular convergent-divergent nozzles. These consist of two shock structures; one originating from the nozzle throat and another from its exit. Analyzing the shock pattern developing for different fluidic injection operating conditions, it is shown that fluidic injection allows the rearrangement of the shock structures relative to each other. Overlapping the two structures caused large pressure oscillations in the exhaust and high amplitudes of shock associated noise, whereas staggering the shock structures mitigated these effects. The screech tone frequency did not change for all injection operating configurations, although the shock diamonds had been shifted drastically with respect to each other. Hence, the screech phenomenon is dominated by the primary shock spacing originating from the nozzle throat.
\end{abstract}

\section{Nomenclature}

$A=$ area $\left(m^{2}\right)$

$c \quad=$ speed of sound $(\mathrm{m} / \mathrm{s})$

$D=\operatorname{diameter}(m)$

$\mathcal{D}=$ linear system matrix

$f_{s}=$ screech frequency $(H z)$

$i^{2}=-1(-)$

\footnotetext{
*Research Associate.

${ }^{\dagger}$ NRC Research Fellow, AIAA Member

$\ddagger$ Distinguished Professor. AIAA Fellow

$\S$ Associate Professor. AIAA Senior Member
} 


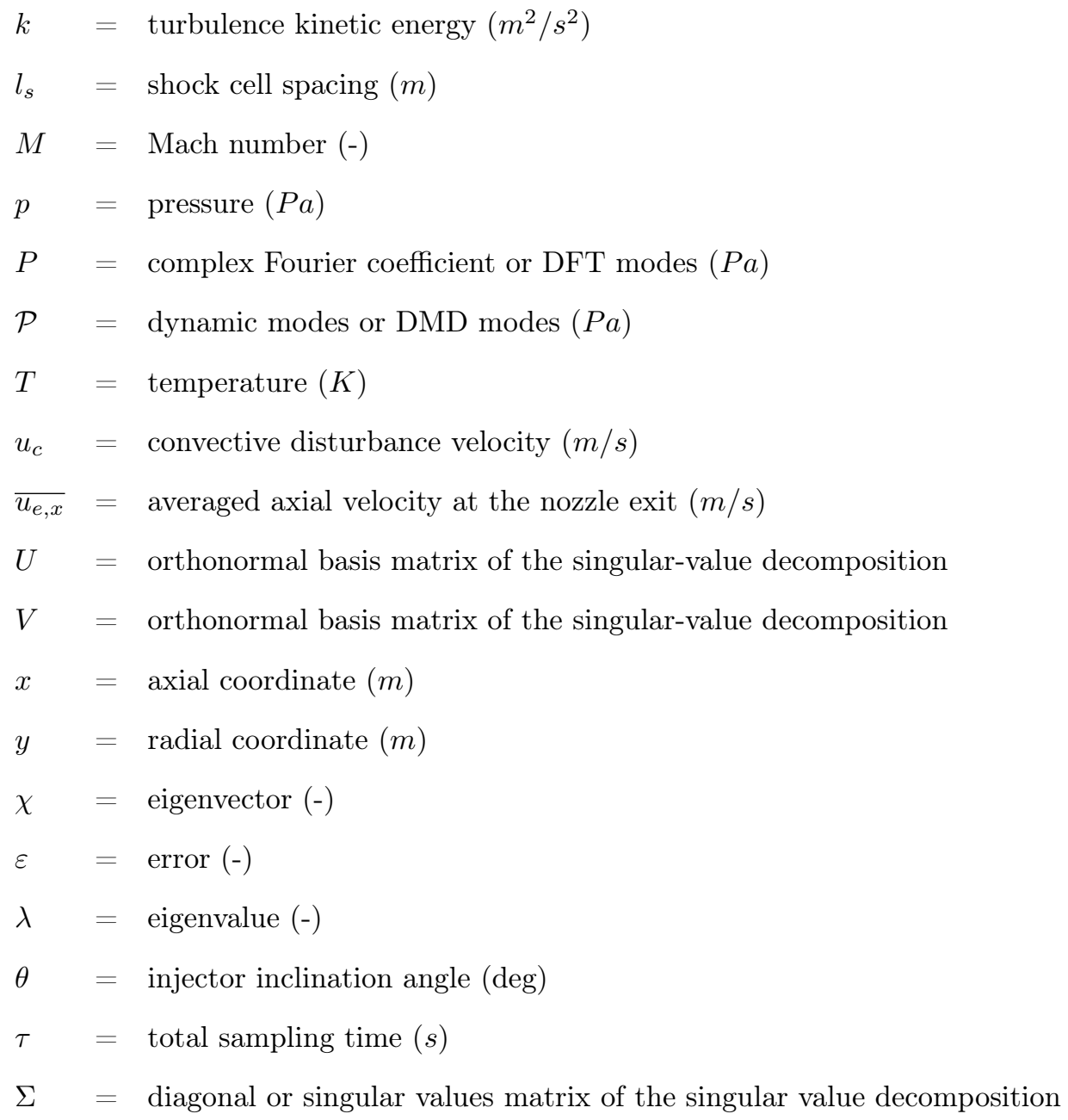

\section{Introduction}

Area adjustable convergent-divergent nozzles of supersonic aircraft have typically sharp contoured throats. This geometrical feature gives rise to the formation of double shock diamond patterns in the exhaust plume. Shocks generate acoustic noise when interacting with the shear layer in free jets. Their acoustic signature commonly dominates in the upstream observer angles. Being subject to legislation, acoustic noise attenuation is demanded. Serrated nozzles have been employed to induce streamwise vortical structures in the vicinity of the nozzle [1]3], which dissipate kinetic energy in the early development of the shear layer and thereby reduce the formation of large flow structures further downstream [4]. Alternatively, fluidic injection provides the advantage of being deactivated when not required [5] 9 . Fluidic injection was found especially efficient to reduce shock associated noise [10. Nonetheless, the design of fluidic injection systems requires parametric studies, because the interaction of the injected flow with complex shock patterns involves non-linear interactions that are difficult to predict [11, 12]. 
Substantial progress has been made in understanding shock noise generation. Suzuki and Lele [13] found numerically that shocks can leak acoustic waves through the shear layer near saddle points of vorticity. Edgington-Mitchell et al. [14] showed by performing statistical analysis of experimental flow visualizations that shock interaction with the shear layer affects the coherence of the vorticity and this might facilitate acoustic waves to penetrate. The importance of shock pattern quasi-periodicity to broadband shock associated noise was highlighted by Harper-Bourne and Fisher [15] as being the prerequisite for constructive interference of acoustic waves generated by the interaction of coherent flow structures and the shocks. Further theoretical and experimental analysis of the broadband shock associated noise has been performed by Tam and Tanna [16], who extended the theoretical model developed by Harper-Bourne and Fisher [15] for convergent nozzles to convergent-divergent nozzles. Tam et al. [17] suggested that screech tones can be interpreted as a special form of broadband shock associated noise, where Berland et al. [18] showed that the lateral displacement of the shocks is essential for screech. Panda [19] noted that the shocks close to the nozzle exhibit sinusoidal motion, whereas the downstream shock cells undergo large displacement while splitting into weaker shocks. During the screech cycle, a new traveling shock is generated upstream of the original shock becoming stronger while the downstream shock disappears [20]. Gojon et al. 21] analyzed the origin and the properties of the oscillation modes in screeching, non-ideally expanded round jets using a jet vortex sheet model. It has been shown that at the screech frequencies, there exist acoustic waves in the jet flow that are propagating in the upstream direction at the ambient speed of sound. It has been suggested that the feedback path of the mechanism causing the screech noise is achieved by such waves.

Powell [22] described screech as feedback loop of flow instabilities convecting in the shear layer and generating acoustic perturbations that interact with the regularly spaced shock train. These acoustic waves travel upstream to the nozzle exciting there the sensitive shear layer to reinforce the initial flow instabilities. Powell [22] used phase and amplitude criteria to estimate the screech frequency, $f_{s}=u_{c} /\left(l_{s} \cdot\left(1+u_{c} / c\right)\right)$, where $u_{c}$ is the convective velocity of the disturbance, $c$ is the speed of sound, and $l_{s}$ is the shock spacing. This simple formula has the drawback that the shock cell spacing and the convective disturbance velocity are difficult to estimate for complex shock pattern structures, such as the double shock diamond pattern. For example, Westley and Woolley [23] showed by detailed Schlieren movies that the disturbances do not travel with a constant convective speed, but are accelerated and decelerated as they interact with the shock train.

Although a general theory of simple shock noise sources could be established, little is known when it comes to more complex shock pattern. Fluidic injection and serrated nozzles have been theorized to attenuate screech by diminishing the shear layer excitation downstream of the nozzle. The present experimental far-field measurements will show that the screech amplitude is found to behave in a non-linear manner with respect to the applied injection pressures. Using detailed numerical simulations, we demonstrate how fluidic injection 


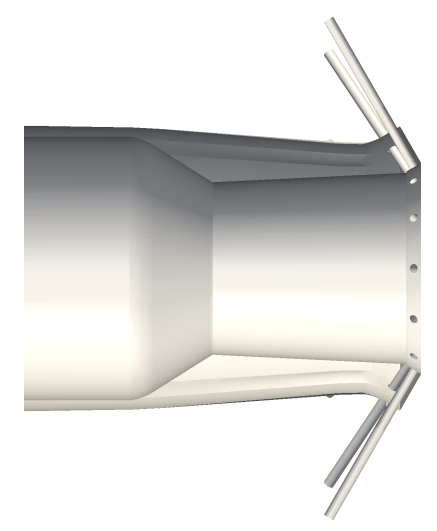

(a) mid-plane side view

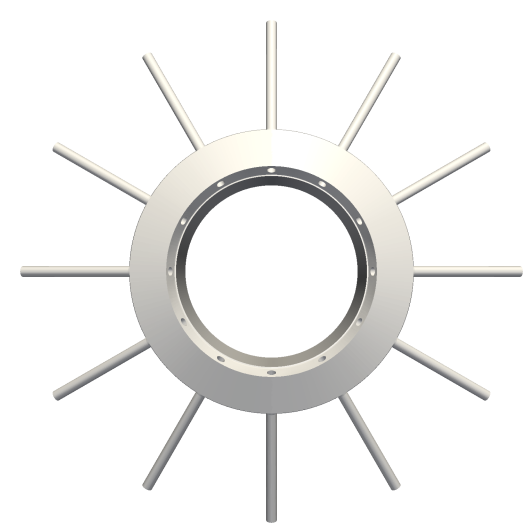

(b) front view

Fig. 1 The investigated convergent-divergent nozzle geometry is shown.

transforms the double shock diamond pattern in convergent-divergent nozzle exhaust plumes and influences thereby the acoustic noise generation. Many investigations of screech and broadband shock associated noise rely on the quasi-periodicity of the shock pattern, while intersecting shock pattern such as double diamonds challenge their theory. We demonstrate that fluidic injection at the nozzle exit displaces the exit shock diamonds (arising from the nozzle exit) with respect to the throat shock diamonds (generated at the nozzle throat). Thereby, the distance between the shock diamonds is varied, and it is shown that the screech tone frequency remains unaltered, being governed by solely by one shock diamond pattern.

\section{Nozzle configuration}

The investigated convergent-divergent nozzle geometry is shown in Fig. 1. The inner nozzle shape is characterized by its area ratio of 1.23 and the design Mach number of 1.56 . The nozzle exit diameter, $D_{e}$, is $0.0575 \mathrm{~m}$. The airflow is driven steadily by a compressor, providing a nozzle total pressure of four times the ambient pressure. The nozzle total temperature is 1.27 times the ambient temperature. The supersonic jet expands into a quiescent ambient medium. For the computational set-up, the ambient pressure, $p_{\infty}$, is set to be $101325 \mathrm{~Pa}$ and the ambient temperature is $288.15 \mathrm{~K}$. Twelve cylindrical injectors are installed equidistantly around the circumference of the nozzle exit, where a small step separates the pipes from the inner nozzle contour. The injectors, with a diameter of $2.7 \mathrm{~mm}$, are inclined $60^{\circ}$ relative to the nozzle's axis. The total temperature at the injector inlet is equal to the ambient temperature. The injected flow is supplied from a plenum, where the injector total pressure, $p_{0, j}$, is controlled as ratio to the ambient pressure, $p_{\infty}$, and will be referred to as the Injection Pressure Ratio (IPR), $p_{0, j} / p_{\infty}$ * The operating conditions are summarized in Tab. 1 .

*The injection pressures 6.6 and 7.8 have only be considered numerically. 
Table 1 Reference values and operating conditions.

\begin{tabular}{llrl} 
parameter & symbol & value & unit \\
\hline nozzle exit diameter & $D_{e}$ & 57.5 & $\mathrm{~mm}$ \\
nozzle area ratio & $A_{e} / A^{\star}$ & 1.23 & $(-)$ \\
nozzle design Mach-number & $M_{e}$ & 1.56 & $(-)$ \\
nozzle pressure ratio & $p_{0, \text { noz }} / p_{\infty}$ & 4 & $(-)$ \\
nozzle inlet temperature & $T_{0}$ & 367 & $\mathrm{~K}$ \\
injector pipe diameter & $D_{j}$ & 2.7 & $\mathrm{~mm}$ \\
injector inclination angle & $\theta$ & 60 & $\circ$ \\
injection pressure ratio & $p_{0, j} / p_{\infty}$ & $1.0,1.8,2.4,3.6,5.5,6.6,7.8$ & $(-)$ \\
ambient pressure & $p_{\infty}$ & 101,325 & $\mathrm{~Pa}$ \\
ambient temperature & $T_{\infty}$ & 288.15 & $\mathrm{~K}$
\end{tabular}

\section{Experimental Approach}

The experimental investigation has been performed in the Aeroacoustic Test Facility GDPL at the University of Cincinnati. This facility is equipped with a coaxial jet test rig situated in an anechoic chamber, which can simulate realistic operating conditions of nozzle pressure and temperature ratios with $1 / 8^{\text {th }}$ scale nozzles. More detailed information of the test facility is provided by [24].

The sound pressure levels $f^{\dagger}$ radiated into the far-field were recorded using 13 microphones. The $1 / 4^{\prime \prime}$ Bruel \& Kjaer model 4954 free-field condenser microphones, with frequency sensitivity up to $100 \mathrm{kHz}$, were placed at a distance of 65 nozzle exit diameters from the nozzle exit. The microphones were distributed at spacings of ten degrees from $40^{\circ}$ to $150^{\circ}$ (where the direction far-downstream, along the jet center-line is considered at 180 degrees). Another microphone was mounted upstream of the nozzle exit plane at $35^{\circ}$. The signals were sampled at a frequency of $204.8 \mathrm{kHz}$ for a period of 15 seconds. A bandpass filter with a lower cut-on margin of $350 \mathrm{~Hz}$ and an upper cut-off frequency of $100 \mathrm{kHz}$ was employed on the dataset. The narrowband frequency spectra were computed using discrete fast Fourier transform's with a window size of 4096 samples, which leads to an ensemble number of 750 realizations and frequency resolution step of $50 \mathrm{~Hz}$.

Particle Image Velocimetry (PIV) measurements are performed using a LaVision PIV system, which consists of a double-pulsed Nd:YAG laser (New wave Research Solo-PIV), and two Imager Intense CCD cameras with an image resolution of $1367 \times 1040$ pixels. Atomized olive oil particles with a diameter of approximately one micrometer have been used for flow seeding. 500 image pairs were sampled with a field view resolution that has been set to $16 \times 16$ for cross-correlations analysis. The data acquisition and vector post-processing have been performed with LaVision software DaVis.

\footnotetext{
${ }^{\dagger}$ The overall sound pressure levels were calculated based on the root-mean-square of the bandpass filtered pressure data $p_{r m s}, O A S P L=20 \cdot \log \left(p_{r m s} / p_{r e f}\right)$, where the reference pressure, $p_{\text {ref }}$, was $20 \mu P a$.
} 


\section{Numerical Method}

The computations consider the compressible Navier-Stokes equations, i.e. conservation equations of mass, momentum, and energy, which are discretized by using a finite volume approach. The viscous stresses are described by Newton's law, and the heat flux is defined by Fourier's law. The flowing medium is air behaving as an ideal gas with an isentropic exponent of 1.4. The dependency of the dynamic viscosity on temperature changes is modeled by Sutherland's formula.

A low-storage four-stage Runge-Kutta scheme was used for time-integration with standard coefficients and a global time-step of $6 \cdot 10^{-9} s$. A second-order central difference scheme has been applied for spatial discretization of the convective terms. A pressure difference triggered Jameson-type artificial dissipation [25] consisting of a blend of second and fourth order differences to prevent spurious oscillations near sharp gradients, e.g. shock waves. The viscous terms in the momentum equations are computed estimating the gradients by a Laplacian formulation and the missing components by a Green-Gauss formulation, which leads to a second-order accurate solution. Adiabatic no-slip boundary conditions have been employed at all solid surfaces. At the inflow and outflow boundaries, characteristic boundary conditions have been applied. The total pressure and total temperature are specified at the inlet, while the static pressure and static temperature corresponding to the ambient quantities are stated as target values at the outlet boundaries.

Resolving all the flow scales of the turbulent jet flow would lead to an excess of computational resources. To keep the required effort within reasonable limits, only a substantial proportion of the kinetic energy decay in the inertial subrange is resolved, which is commonly known as Large Eddy Simulation (LES). Generally, discretization schemes with low dissipative error, such as the central difference schemes, are desired for an accurate simulation of the turbulent energy cascade. The spatial filtering of the governing equations causes additional terms representing the unresolved, so-called subgrid-scales, which require modeling. Careless employment of subgrid-scale filters, models, and constants can influence significantly the flow-field and the acoustic noise radiation [see e.g. 26, 27]. The truncation error of the commonly employed discretization schemes is of the same order of magnitude as the subgrid-scale model contribution, which requires explicit spatial filtering of the governing equations to retain accuracy. Distant from solid walls, the kinetic energy decay at the small-scale turbulence exhibits universal behavior, which supports possible modeling. Implicit subgrid scale models are based on the inherent dissipation of the numerical scheme. The mathematical foundation of this implicit LES approach has been rigorously provided by [28]. [28] show that the numerical truncation error can be rewritten as a Clark subgrid-scale model in the momentum conservation equation and as a Smagorinsky-type subgrid-scale model for the energy equation for a finite volume discretization with a non-oscillatory scheme of at least second order accuracy. Besides, with this approach, the kinetic energy is absolute decrease, and no energy buildup at the high-frequency flow scales occurs. This implicit 
LES approach used in the present simulations has the advantage that no calibration constants are required and the entire information resolved on the mesh is utilized without truncation by an explicit filter, which makes this approach highly computationally efficient.

The computational domain extends over eight nozzle exit diameters radially and 30 nozzle exit diameters in the axial direction. A block-structured grid with 485 blocks and approximately 48 million hexahedral cells is used. Each injector is equipped with its own O-structure to provide a high-quality wall mesh at all locations and immediately downstream of the injector orifice. The near-wall region has been resolved as finely as possible, where the smallest cell close to the wall has a height of $5 \cdot 10^{-9} \mathrm{~m}$. The general structure can be subdivided into a fine resolved investigation part covering the hydrodynamic near field of the supersonic jet and the inner nozzle region, and sponge zones with a low gradual grid stretching towards the inlets and outlets.

\section{A. Validation}

The numerical approach is validated against experimental PIV data. In contrast to the simulations, a bypass flow Mach number of approximately 0.1 is required to seed the entrained flow for the PIV measurements. Furthermore, the PIV measurements capture only two components of velocity and hence, the radial velocity fluctuations are included twice in the turbulence kinetic energy estimation replacing the non-available azimuthal component. Hence, differences are expected.
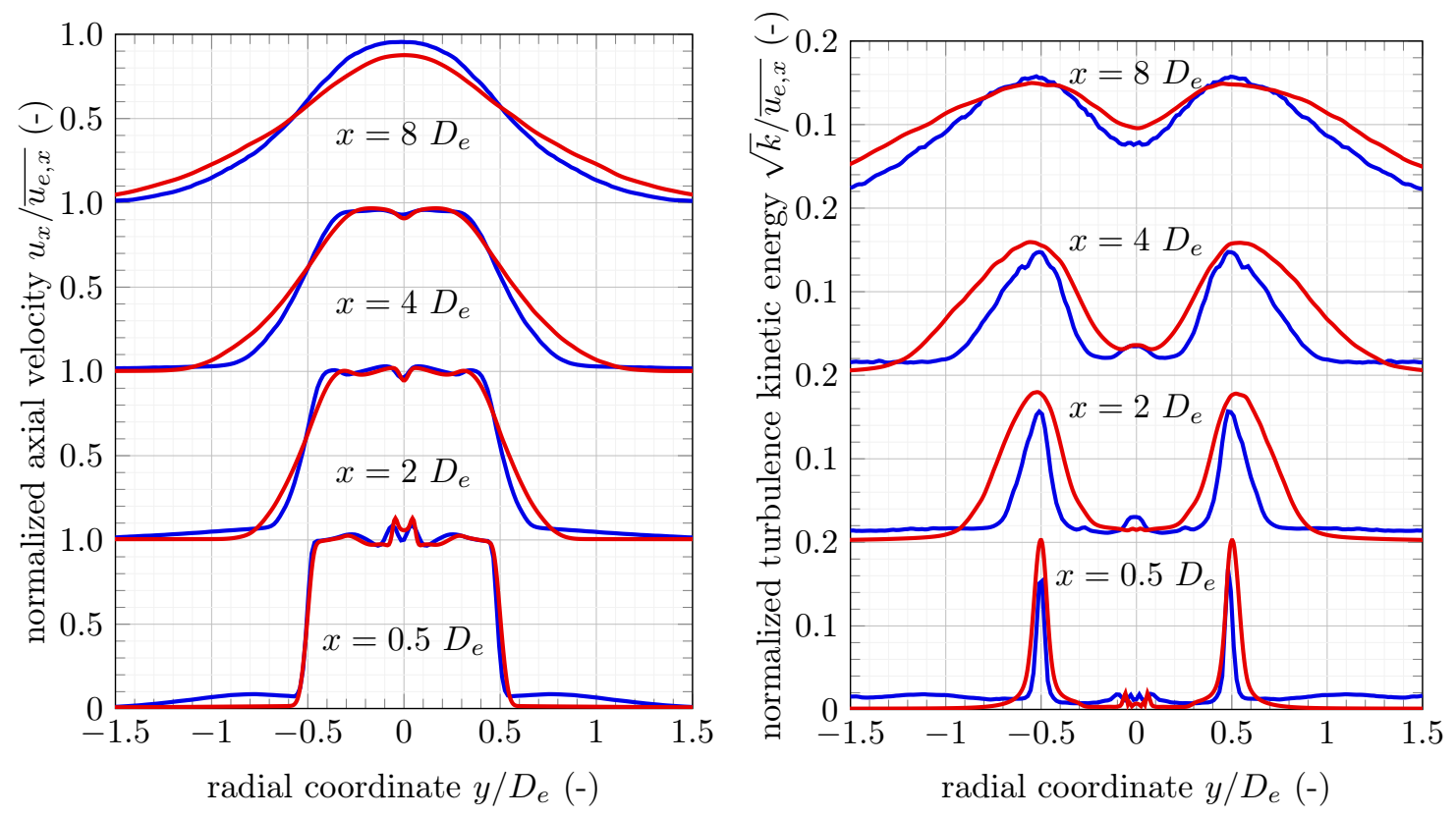

Fig. 2 Without fluidic injection, the numerical simulation results (red) are compared with experimental PIV measurements (blue) in terms of the axial velocity component and the normalized turbulent kinetic energy. 
As shown in Fig. 2, the simulations capture the exhaust behavior in good agreement with the experiments. The initially higher turbulence kinetic energy levels in the vicinity of the nozzle, predicted by the simulations, are consistent with the larger velocity gradient predicted across the shear-layer due to the missing bypass flow. This might have an impact on the turbulent kinetic energy levels right downstream of the nozzle.

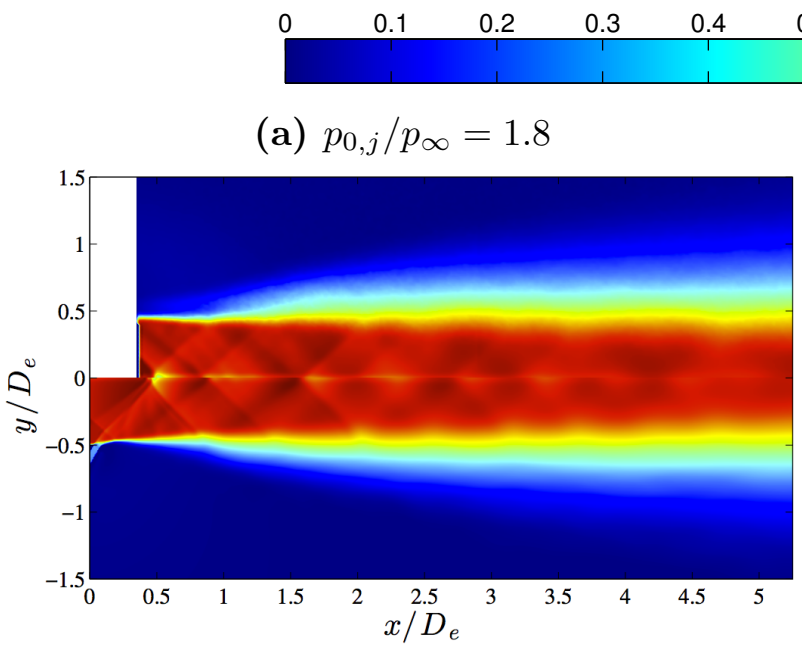

(c) $p_{0, j} / p_{\infty}=3.6$
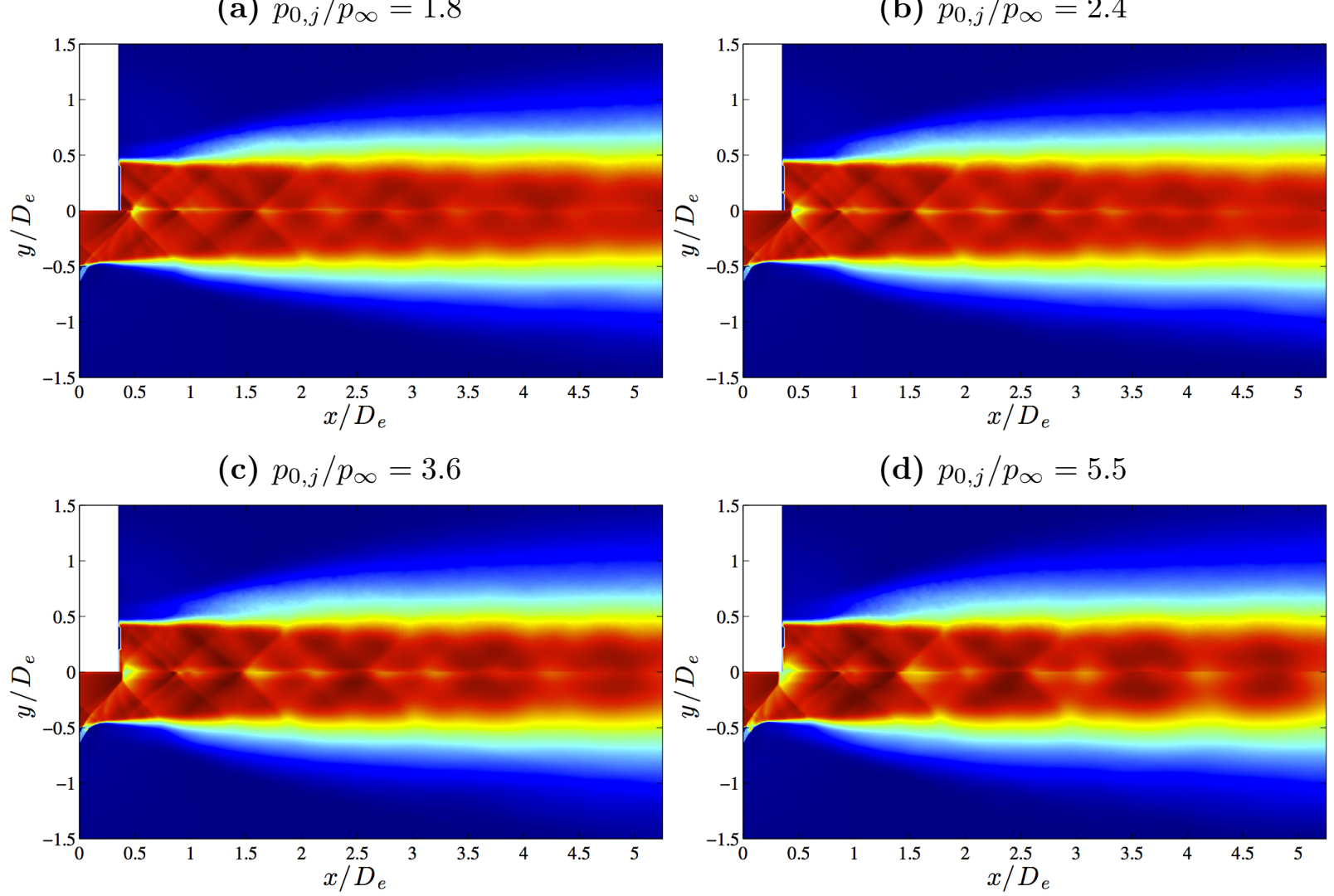

(d) $p_{0, j} / p_{\infty}=5.5$

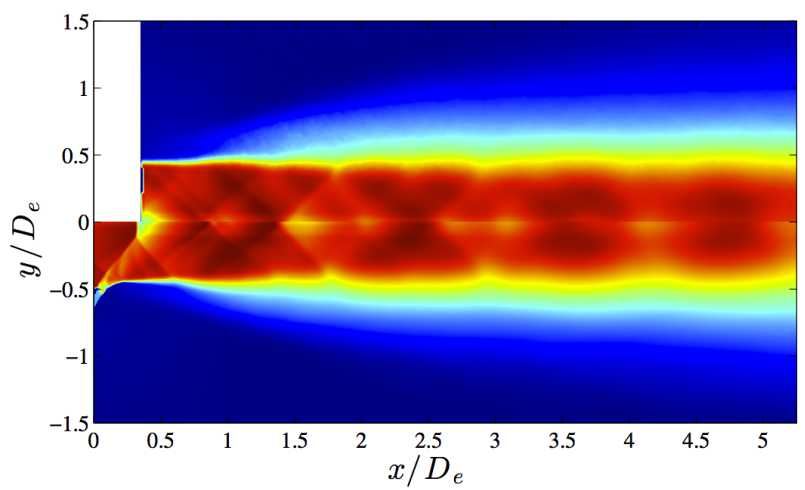

Fig. 3 The experimentally (top half) and numerically (lower half) estimated time-averaged axial velocities normalized by the averaged nozzle exit velocity are compared in a mid-plane view.

Figure 3 shows that shock wave locations, angles and slip lines are reproduced accurately for all operating conditions of fluidic injection. The normalized axial velocity contours are in excellent agreement. Differences can be observed comparing the turbulence kinetic energy contours assessed experimentally and computationally in Fig. 4, which consist mainly in the fluctuation amplitudes captured in shear layer 2.5 to 4.5 nozzle exit diameters downstream of the nozzle exit and fluctuations captured outside of the shear layer. The latter flow disturbances occur due to the low seeding density and low particle displacement in the bypass flow. It is worthwhile to note that both approaches reveal consistently an abrupt decrease of the turbulence kinetic 
energy where shocks interact with the shear layer after about $x=2 D_{e}$ downstream.

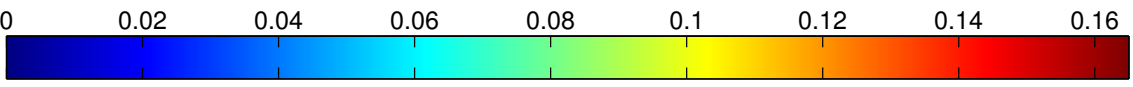

(a) $p_{0, j} / p_{\infty}=1.8$

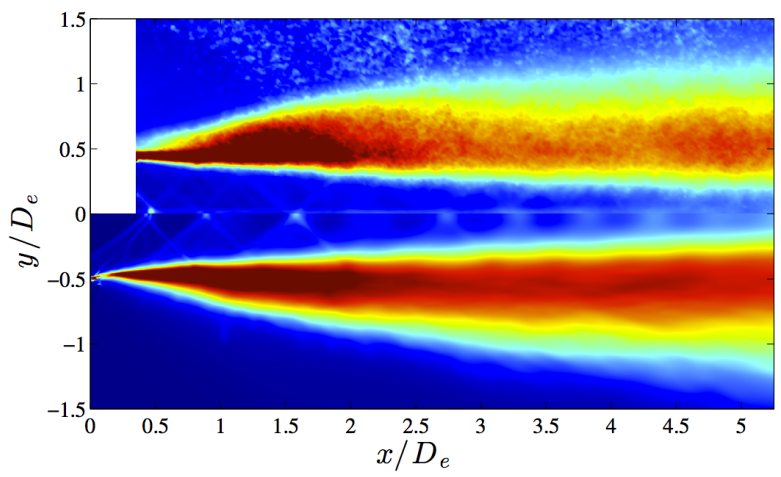

(c) $p_{0, j} / p_{\infty}=3.6$

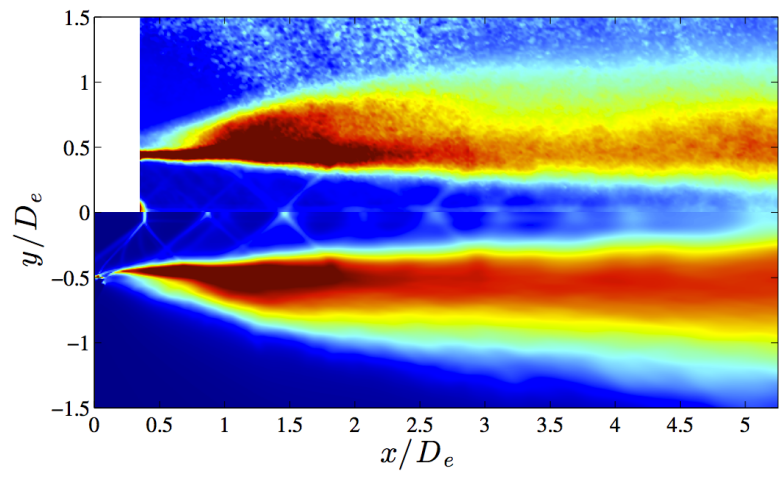

(b) $p_{0, j} / p_{\infty}=2.4$

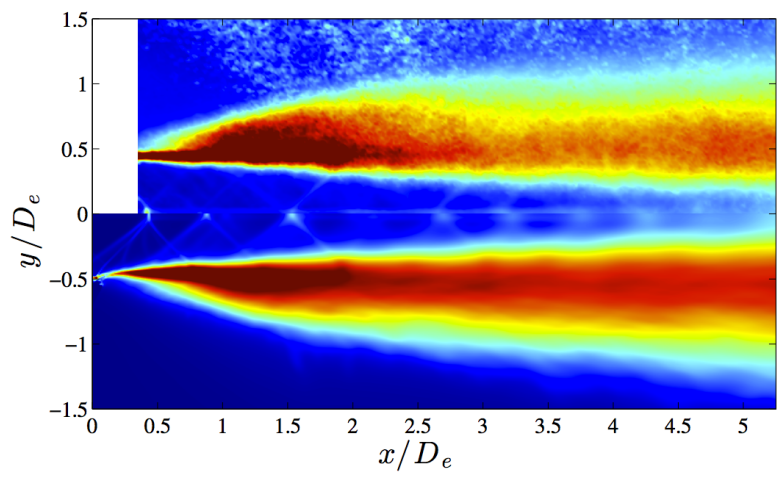

(d) $p_{0, j} / p_{\infty}=5.5$

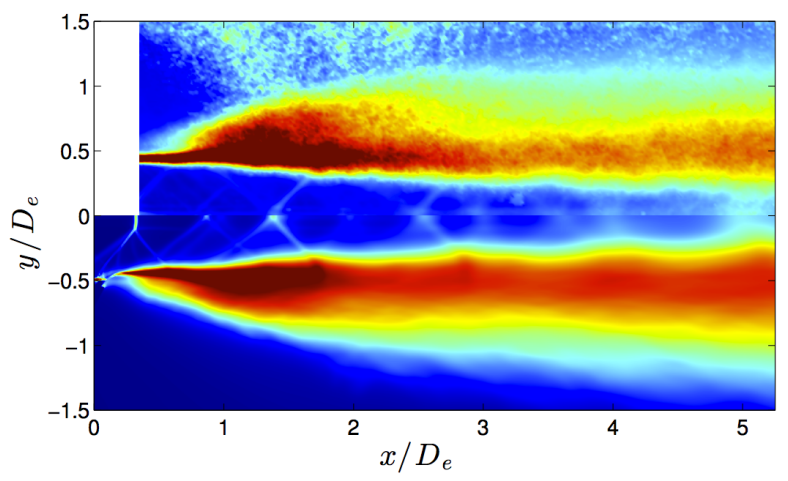

Fig. 4 Normalized turbulence kinetic energy, $\sqrt{k} / \overline{u_{e, x}}$; experimental particle image velocimetry data is shown in the top half and computational results are plotted on the lower half.

Despite the differences in the setup, the relative error of the turbulence kinetic energy, i.e. $\varepsilon=\mid k_{P I V}-$ $k_{L E S} \mid / k_{P I V}$, remained below $30 \%$ in the shear layer while the shock locations have been accurately captured. Hence, a good agreement between experimental PIV measurements and the numerical simulations could be achieved in the region of the exhaust.

\section{Acoustic Far-Field}

Figure 5 (d) demonstrates the measured acoustic noise reduction trend, where intermediate injection pressures achieve the most favorable attenuation (except at the furthest downstream receiver angles, where high injection pressures are beneficial). At the downstream receiver angles, the turbulent mixing noise, resulting from the large flow scales, dominates with the highest sound pressure levels measured in the far field. The screech tones can be identified for all injection configurations in the upstream receiver angles (shown in 
Fig. 5 (a) and (b)) at a frequency of approximately $2.18 \mathrm{kHz}$ and the shock associated broadband noise can be noted in the range of $3-10 \mathrm{kHz}$. The screech tone and shock associated broadband noise amplitude are the lowest for an injection pressure ratio of 2.8 , while being higher with less or more injection pressure.
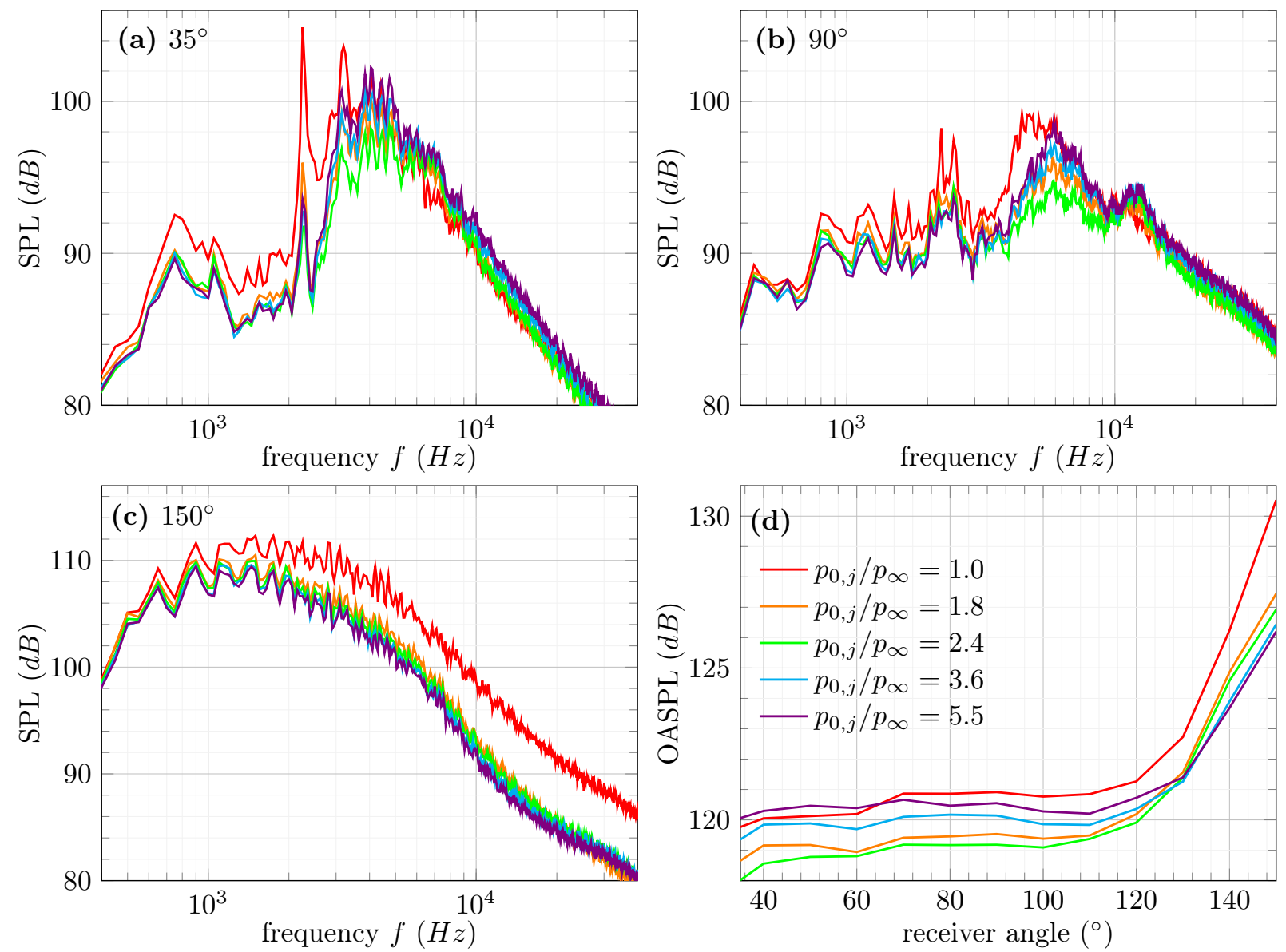

Fig. 5 Frequency characteristic of the acoustic noise measured in the far-field at three receiver angles (a-c) and overall sound pressure levels, (OASPL), (d).

\section{Exhaust Near-Field}

Figure 6 shows that a shock wave is initiated downstream of the nozzle throat and it focuses (as Mach disk) on the centerline. The oblique shock wave reflection interacts with the exhaust shear-layer downstream of the nozzle exit, where an expansion fan develops. This shock structure emanating from the nozzle interior forms the throat shock diamond pattern (consisting of alternating expansion fans and shock waves). Without injection, a second shock structure occurs at the nozzle exit due to the slight over-expanded operating condition. The oblique shocks merge to form another Mach disk initiating a second, exit shock diamond pattern. Figure 6 exhibits a few double shock diamonds until they become unrecognizable towards the end of the potential core in the time-averaged visualizations. 


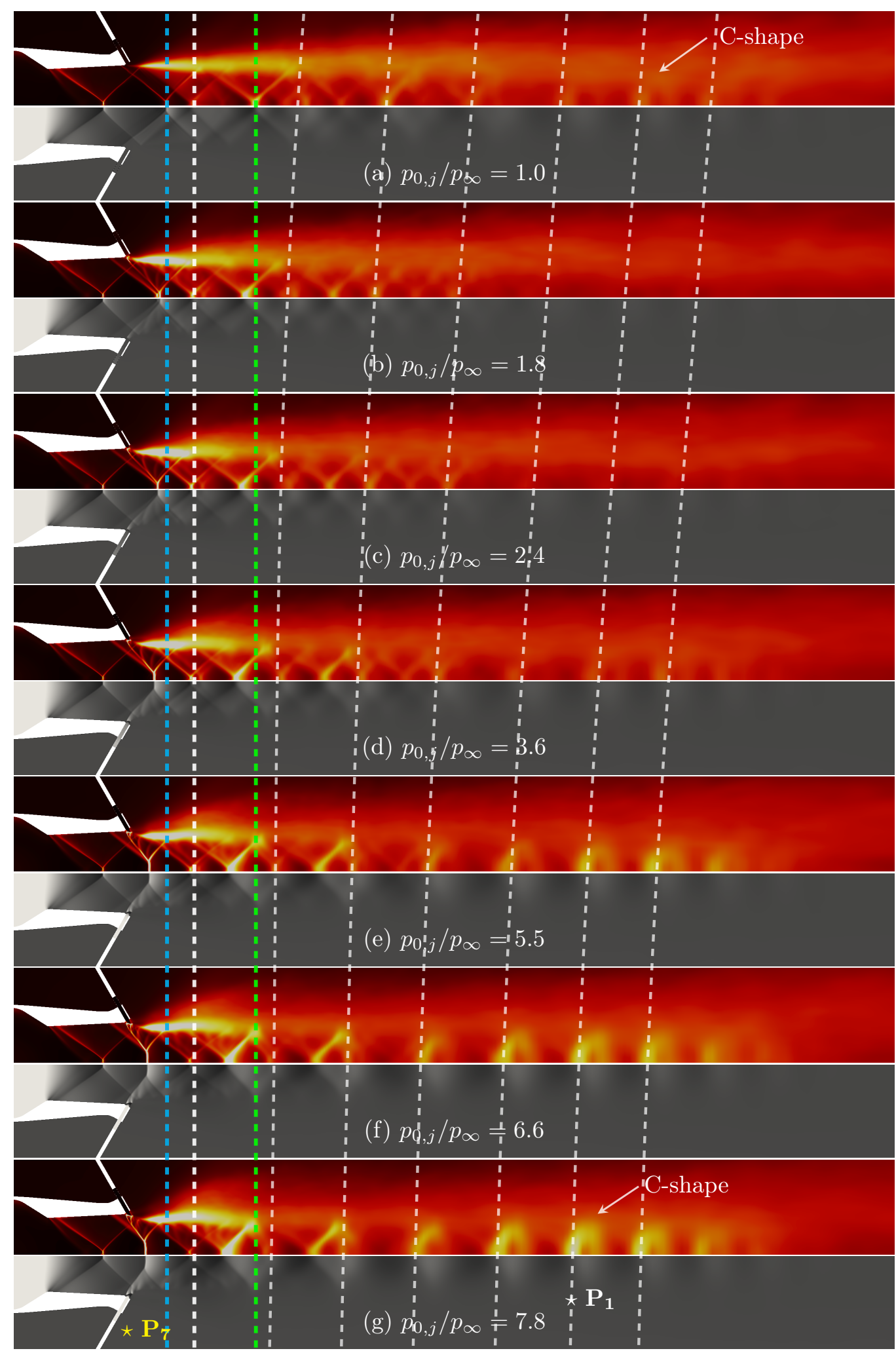

root-mean-square of the static pressure $p_{r m s}(\mathrm{~Pa})$

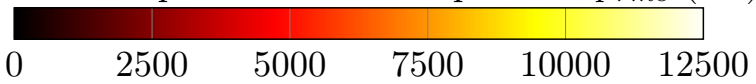

static pressure $p_{s}(k P a)$

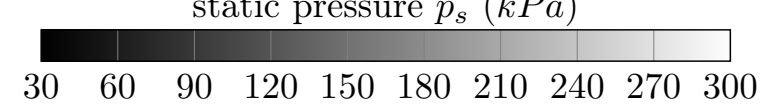

Fig. 6 The static pressure fluctuations and time-averaged static pressure are shown in-plane with the injectors. 
Injection partially blocks and diverts the nozzle exhaust plume around the injected flow. The momentum exchange between the injected jet and the nozzle stream induces high pressures upstream of the interaction point. Hence, bow shocks are established upstream of the injection displacing the shocks off the nozzle exit as shown in a detail in Fig. 7. Increasing the injection pressure leads to deeper jet penetration and hence more blockage with higher pressures upstream of the injection. Due to the higher pressures downstream of the oblique shocks, the shock angles of the exit pattern steepen. The Mach disk shifts upstream and widens (due to the lower Mach-numbers), as observable following the blue dashed line in Fig. 6 marking the Mach disk location. The green dashed line shows the upstream shift of the entire first, exit shock cell. The throat shock pattern location is labeled by a white dashed line in Fig. 6, indicating that its position is not affected by fluidic injection. (The consecutive diamonds of the throat shock pattern are slightly shifted upstream as shown by the consecutive white dashed lines in Fig. 6]) Hence, the diamonds of the throat and the exit shock patterns are shifted with respect to each other by the fluidic injection.

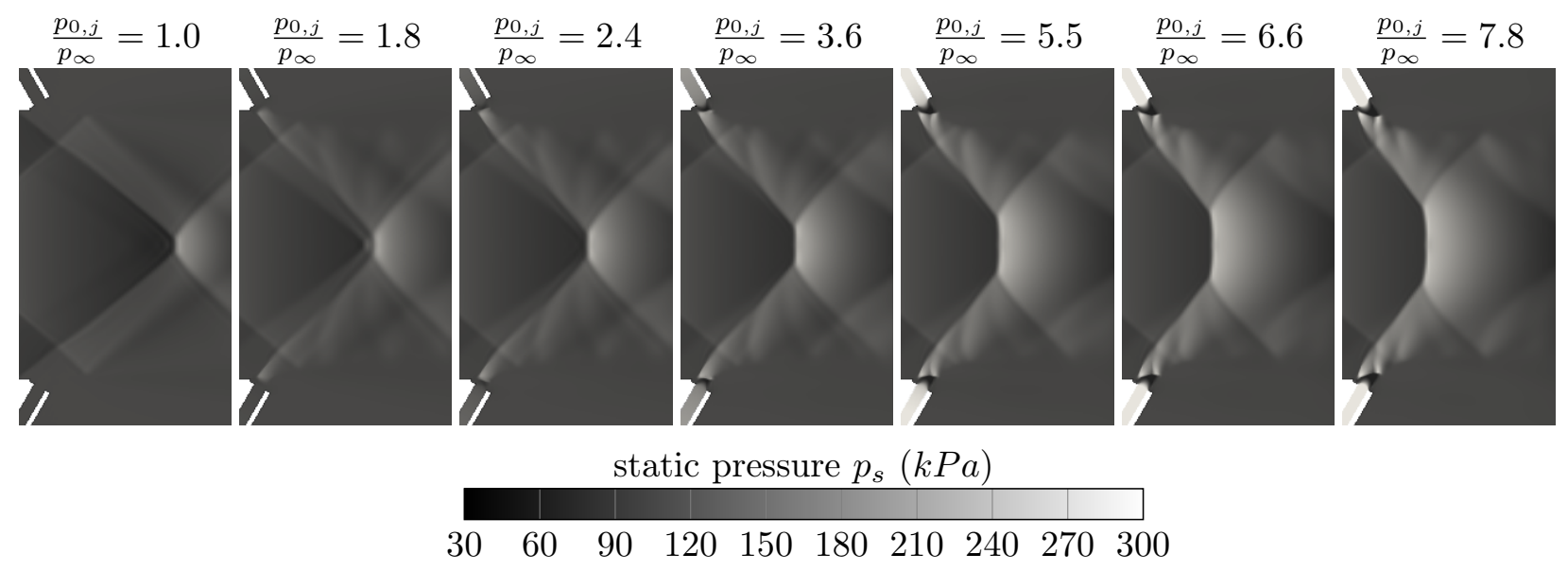

Fig. 7 Detail view of the time-averaged static pressure near the injectors.

The time-averaged pressures on the centerline (shown in Fig. 8) reveal the impact of shifting the exit shock diamonds with respect to the throat shock diamonds. Overlapping the two shock diamonds causes high amplitude pressure oscillations in the exhaust plume with one crest and one valley per overlapped diamond. By intersecting the shock diamonds, two peaks and two valleys of lower amplitude are induced.

Pressure fluctuations are caused by the movement of shocks and the unsteady flow in the shear layers. The amplification of the time-averaged pressure fluctuations due to shock wave displacement is proportional to the pressure difference over the discontinuity and thus the shock strength. As shown in Fig. 6, the shock waves closing the initial shock cell emerging from the nozzle exit exhibit the highest root-mean-square of the 


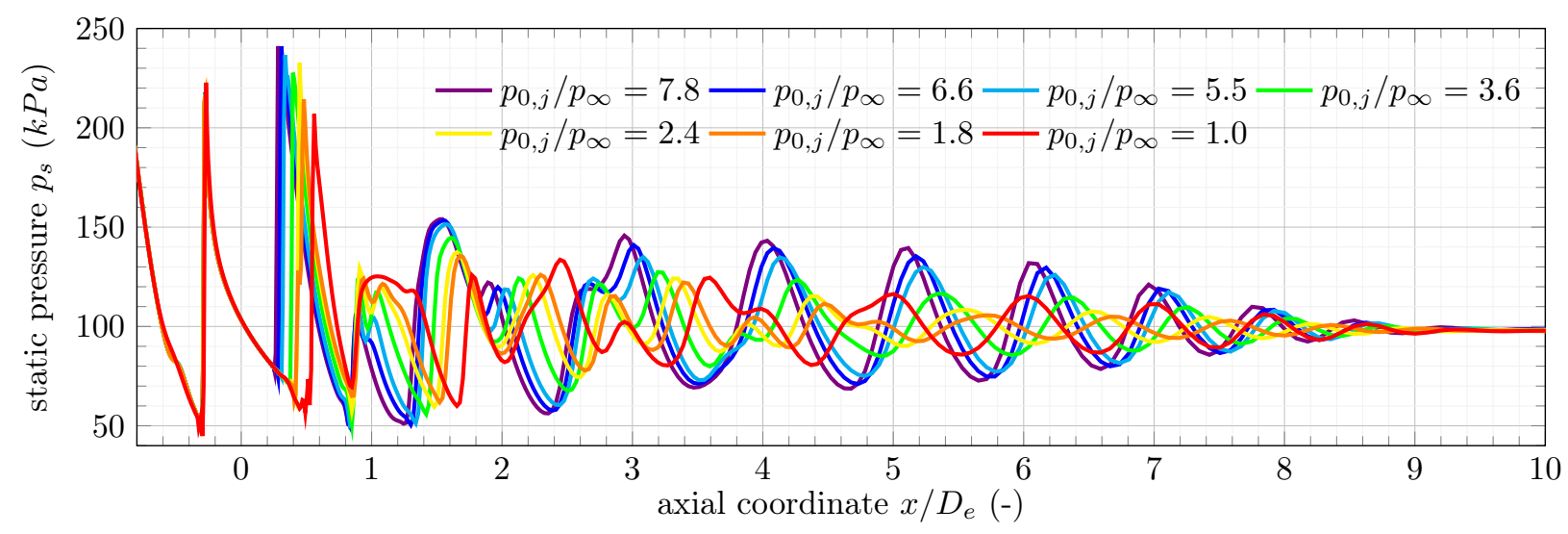

Fig. 8 Time-averaged static pressures along the centerline.

pressure fluctuations, i.e.

$$
p_{\text {rms }}=\sqrt{\frac{1}{\tau} \sum_{t=1}^{\tau}\left(p(t)^{2}-\bar{p}^{2}\right)},
$$

where $t$ is the time, $\tau$ is the total sampling time, and the over-bar denotes the time-average. While the shock waves in the vicinity of the nozzle undergo minimal motion and cause therefore narrowly distributed pressure fluctuations, the lateral motion of the shocks towards the end of the potential core blurs out the pressure fluctuations. Towards the end of the potential core of the jet, these pressure fluctuations appear as C-like shapes without injection and for high injection pressures, as pointed out in Fig. 6. Tracing the shock train responsible for the $\mathrm{C}$-shape pattern back to its origin reveals that they belong to the continuation of the throat shock diamond pattern. (This can be best seen by identifying the throat pattern at intermediate injection pressures and following the trend, which is indicated by white dashed lines, to higher injection pressures.)

\section{Streamwise Vortical Structures}

The blockage of the nozzle exhaust area by the fluidic injection forces the exhaust plume to deflect partially radially outwards [29, see e.g.]. These so-called jetlets can be noted in Fig. 9 from a half to one-and-a-half nozzle exit diameters downstream. This radial outward motion in between injection generates the dominant large-scale counter-rotating vortex pairs, which are shown on radial planes in Fig. 10. It can be observed that the radial extends of these counter-rotating vortex pairs scales the injection pressure. Coherent counter-rotating vortex pairs persist approximately until two nozzle exit diameters downstream of the nozzle exit independently of the injection operating condition. Figures 4 and 9 exhibit an abrupt decay of high turbulence kinetic energy levels when the counter-rotating vortex pairs interact with the shock wave. Consistently with the observation by Edgington-Mitchell et al.[14, the fluctuations are locally enhanced with 
the interaction of shock waves with the shear layer, while decreasing rapidly downstream of such an event.

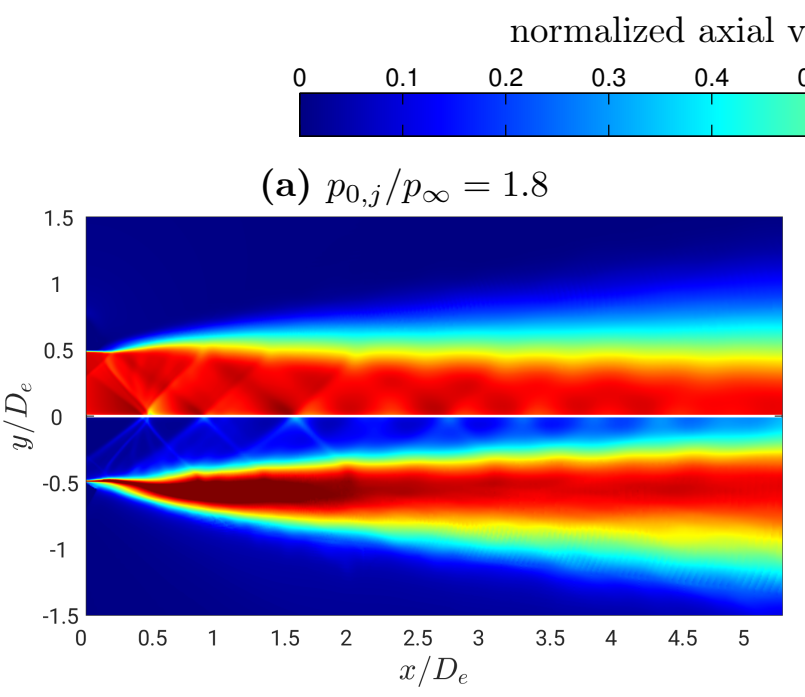

(c) $p_{0, j} / p_{\infty}=3.6$
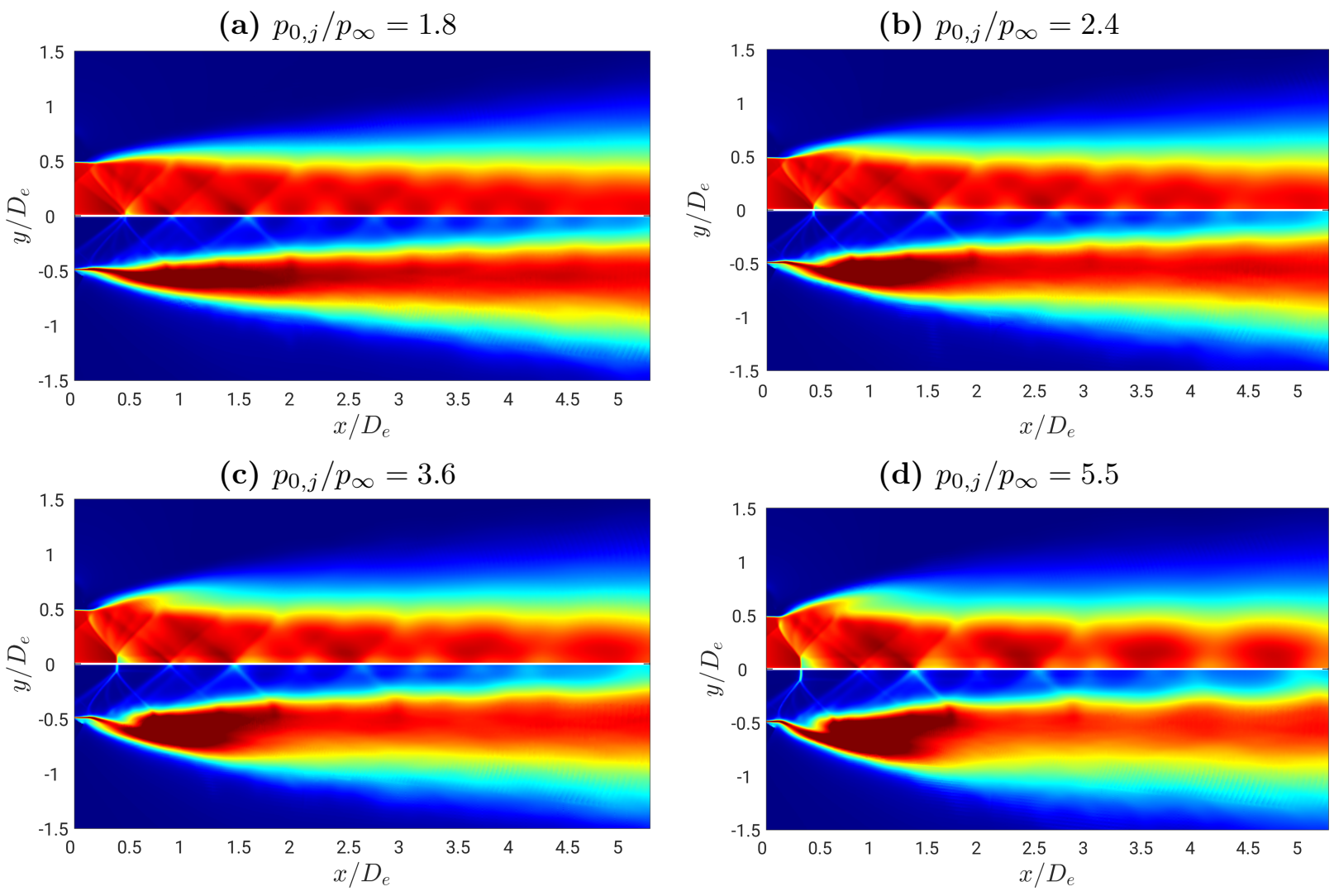

(d) $p_{0, j} / p_{\infty}=5.5$

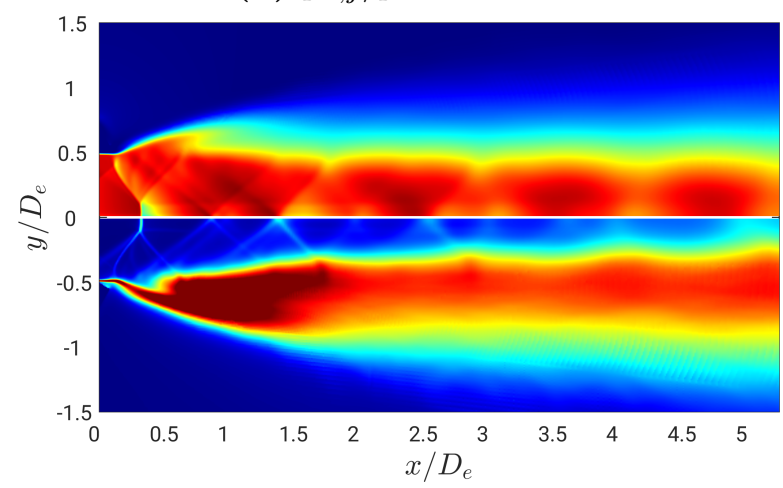

normalized turbulence kinetic energy $\sqrt{k} / \overline{u_{e, x}}(-)$

\begin{tabular}{lllllllll}
0 & 0.02 & 0.04 & 0.06 & 0.08 & 0.1 & 0.12 & 0.14 & 0.16 \\
\hline & 1 & 1 & 1 & 1 & 1 & & \\
\hline
\end{tabular}

Fig. 9 The normalized axial velocity (top half) and the normalized turbulence kinetic energy (lower half) are shown in the plane between injectors.

\section{A. Flow Structures Associated with Screech}

The pressure spectra presented in Fig. 11 exhibit a general broadband distribution with a particular tonal peak at the screech frequency of $2.18 \mathrm{kHz}$ for all injection pressure ratios. The locations of the probes are indicated in Fig. 6. The tonality is the weakest for intermediate injection pressures, as highlighted by the plot at the top of Fig. 11, showing the tonal amplitudes only for the screech frequency. The plot also shows that the estimated screech amplitudes match the experimentally observed trend in the far field. 


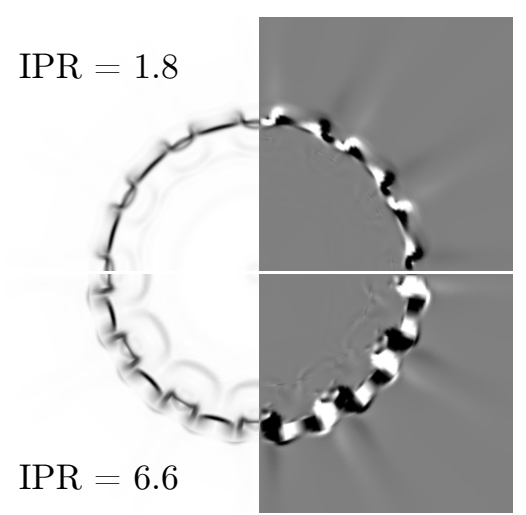

(a) $x=0.1 D_{e}$

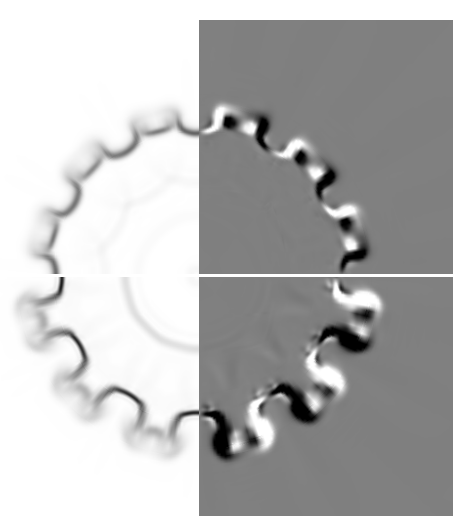

(b) $x=0.2 D_{e}$

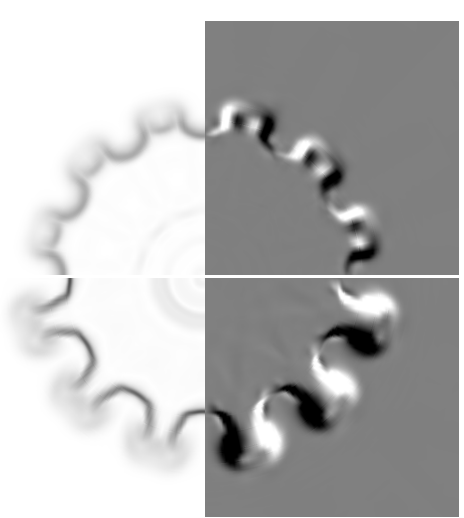

(c) $x=0.3 D_{e}$

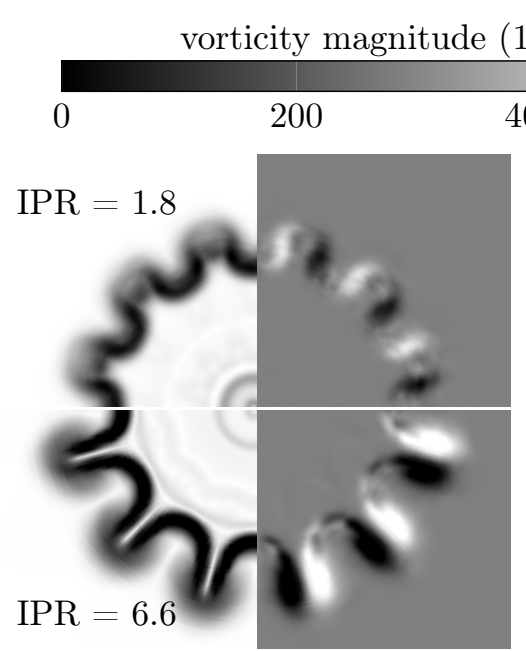

(d) $x=0.5 D_{e}$ (e) $x=D_{e}$

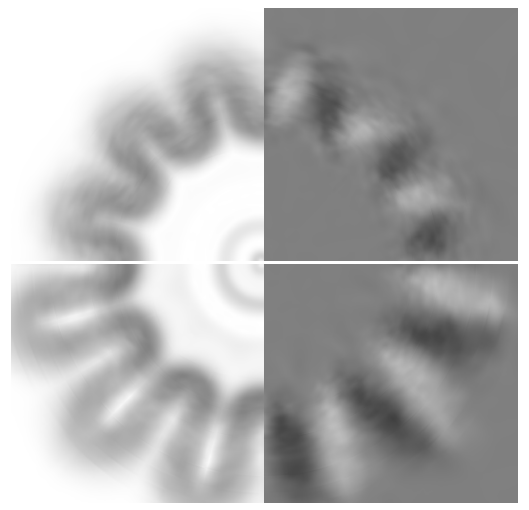

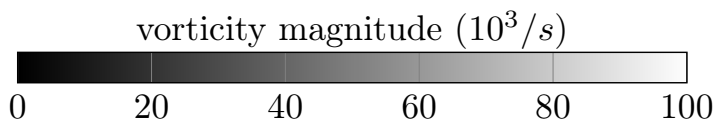

Fig. 10 The vorticity distribution by the fluidic injection is shown in terms of the vorticity magnitude (left half) and the streamwise vorticity component (right half). (The twelve injectors are located at the same locations as the numbers on the clock.)

The tonal amplitude distribution can be analyzed by performing a discrete Fourier transform (DFT),

$$
P\left(f_{j}\right)=\sum_{n=0}^{N-1} p_{n} \cdot e^{-i 2 \pi j n / N}, \quad \text { where } \quad f_{j}=j \Delta f
$$

of the three-dimensional near-field of the exhaust, where $n$ is the index of the sample, $N$ is the total count of samples, and $\Delta f=1 / \Delta t$ is the frequency resolution. 1166 instantaneous pressure field realizations, $p_{n}$, were sampled at a time interval, $\Delta t$, of $5 \cdot 10^{-5} s$ for an injection pressure ratio of 7.8 . (Hence, the highest frequency captured is $10 \mathrm{kHz}$ and the lowest frequency is $17.15 \mathrm{~Hz}$.) The contours of the DFT magnitudes, defined as $\left|P\left(f_{j}\right)\right| / N=\sqrt{\operatorname{Re}\left(P\left(f_{j}\right)\right)^{2}+\operatorname{Im}\left(P\left(f_{j}\right)\right)^{2}} / N$, are shown in Fig. 12 for the screech frequency. The 

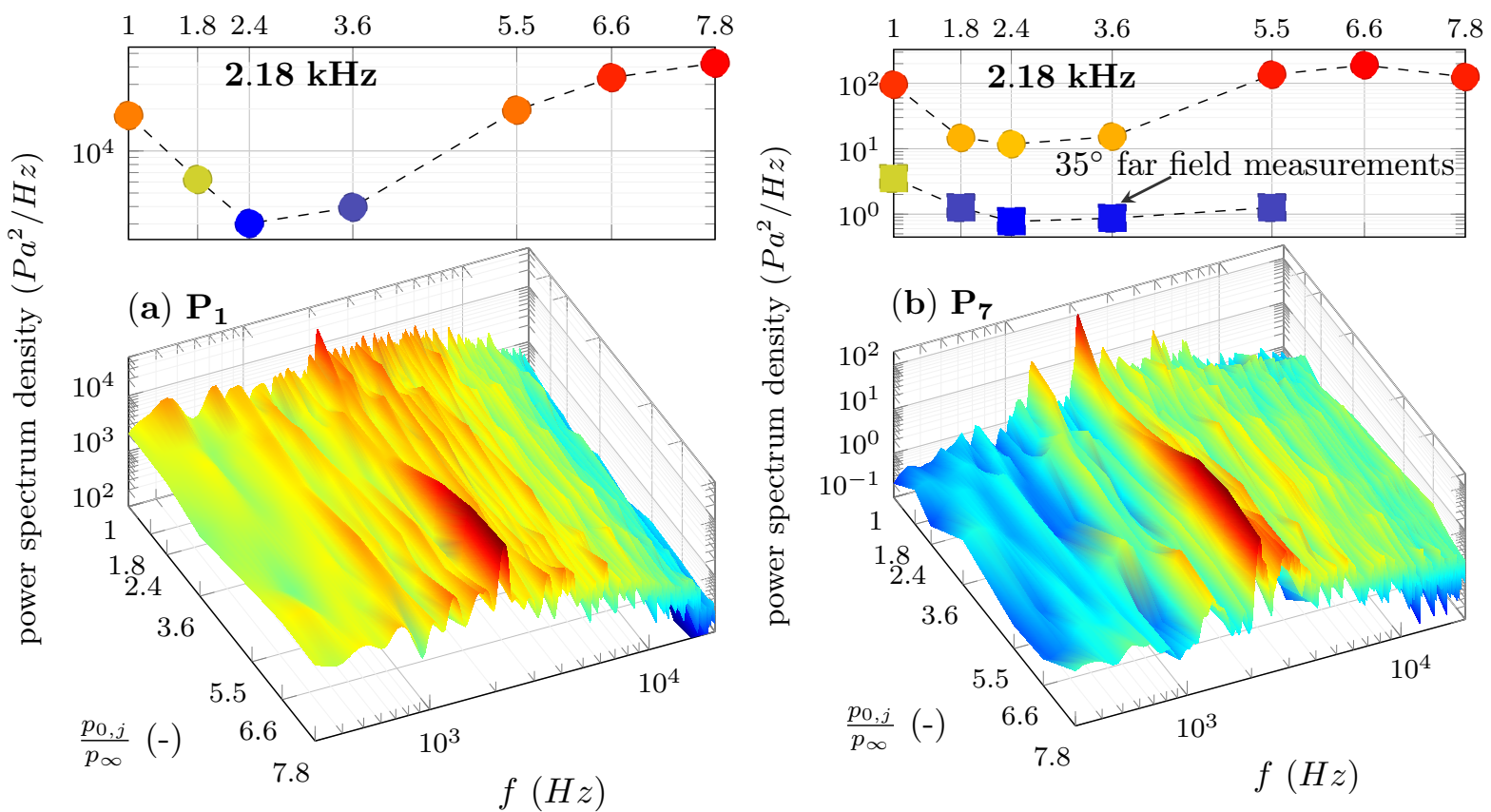

Fig. 11 Spectra obtained in monitoring probes placed immersed in the shear layer (a) and in the acoustic near-field (b).

highest fluctuation amplitudes can be observed where the shocks (of the $4^{\text {th }}$ and $5^{\text {th }}$ shock cell) interact with the exhaust shear layer, which coincides with the locations where the C-shapes of accumulated pressure fluctuations are observable in Fig. 6. The DFT phase angle, defined as $\arg \left(P\left(f_{j}\right)\right)=-i \cdot \ln \left(P\left(f_{j}\right) /\left|P\left(f_{j}\right)\right|\right)$, indicates the propagation speed and direction of the information. Starting from the second shock cell, a phase decay of $2 \pi$ can be observed over each shock cell, which implies that the perturbations convecting in the shear layer interact in-phase with the shock pattern, but do not propagate at a constant speed. Worthwhile to note is that the phase decay changes to a phase increase towards the end of the potential core. [30] linked this observation made in experimental schlieren visualizations to the origin of the screech related perturbations. Fig. 12 shows that this location does not coincide with the distribution of the highest DFT magnitudes.

The perturbations can be reconstructed using the complex Fourier coefficients, $P\left(f_{j}\right)$,

$$
p_{n}=\frac{1}{N} \sum_{j=0}^{N-1} P\left(f_{j}\right) \cdot e^{i 2 \pi j n / N}=\frac{1}{N} \sum_{j=0}^{N-1} P\left(f_{j}\right)(\cos (2 \pi j n / N)+i \sin (2 \pi j n / N))
$$

The corresponding iso-contours are shown in Fig. 13 for the screech frequency, where a double row alternating pattern is exposed. This represents the lateral displacement of the jet core towards the shear layer. In the vicinity of the nozzle, the turbulent mixing induced by the injection masks these structures. Equation 3 states that the real and imaginary component lag each other by a quarter of the screech cycle (which can 


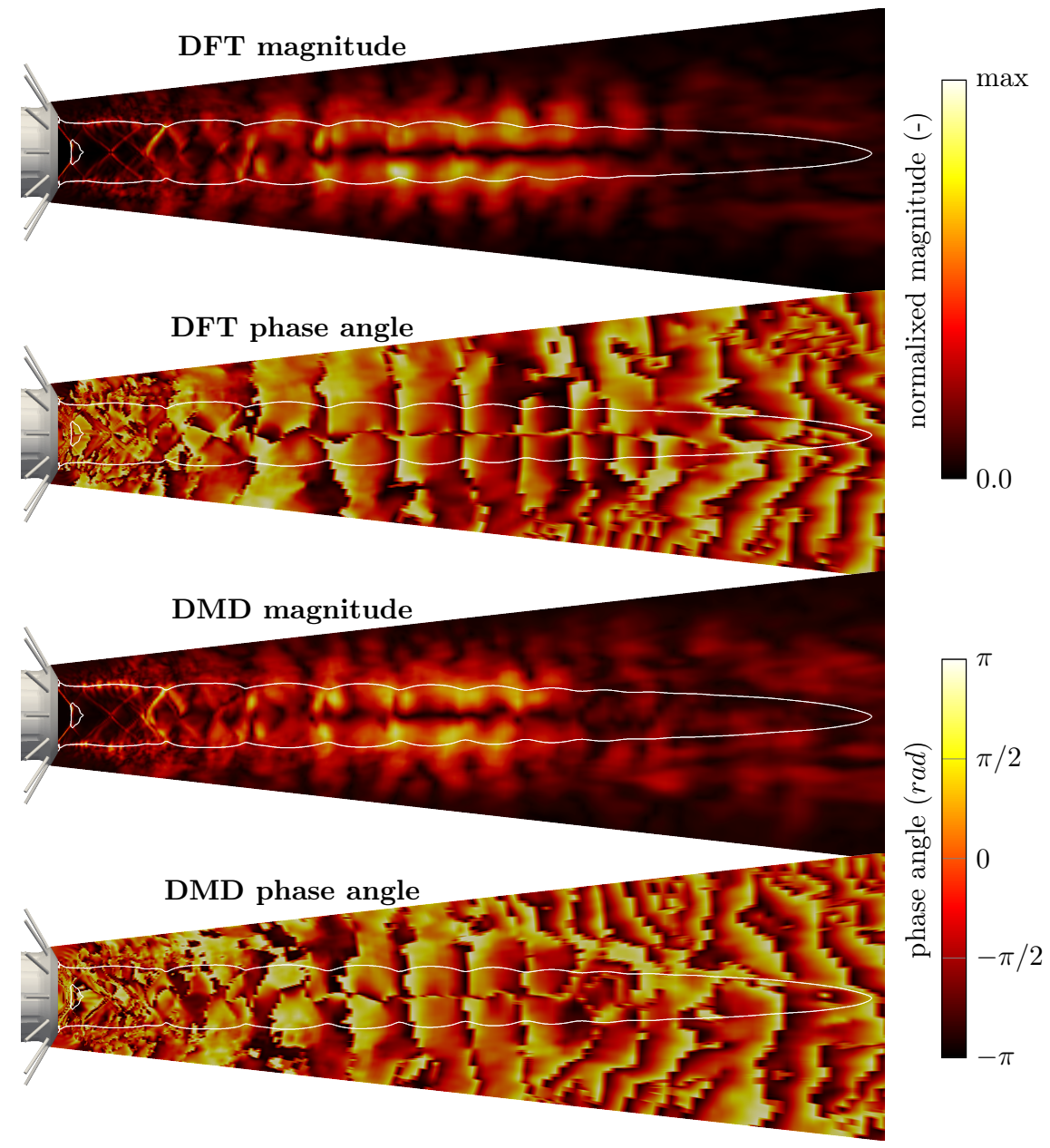

Fig. 12 The magnitude and the phase of the DFT and DMD analysis at the screech frequency is shown in the jet's mid plane for an injection pressure ratio of 7.8. (The white iso-contour indicates the supersonic region, $\bar{M}=1$.)

be noted by the sine and cosine relation). Thus, the propagation of the shapes can be followed in steps of a quarter of the screech cycle in Fig. 13. Although the threshold value for the iso-surfaces was kept equal, the axial extend of the structures is larger in the imaginary component of the DFT mode. This denotes the acceleration and deceleration of the structures before and after the shocks, respectively.

The behavior of a dynamical system is characterized by its eigenvalues and eigenvectors. The dynamic mode decomposition (DMD) technique can be used to extract such features from the given data series, $p_{1}^{N}=\left\{p_{1}, p_{2}, p_{3}, \ldots, p_{N}\right\}$, which is the same as the one used for the DFT analysis. Therefore, a linear tangent operator, $\mathcal{D}$, is assumed to describe the relationship

$$
p_{2}^{N}=\mathcal{D} p_{1}^{N-1},
$$

where $p_{1}^{N-1}$ and $p_{2}^{N}$ contain the first $N-1$ and last $N-1$ entries of $p_{1}^{N}$ respectively. Performing a singular 


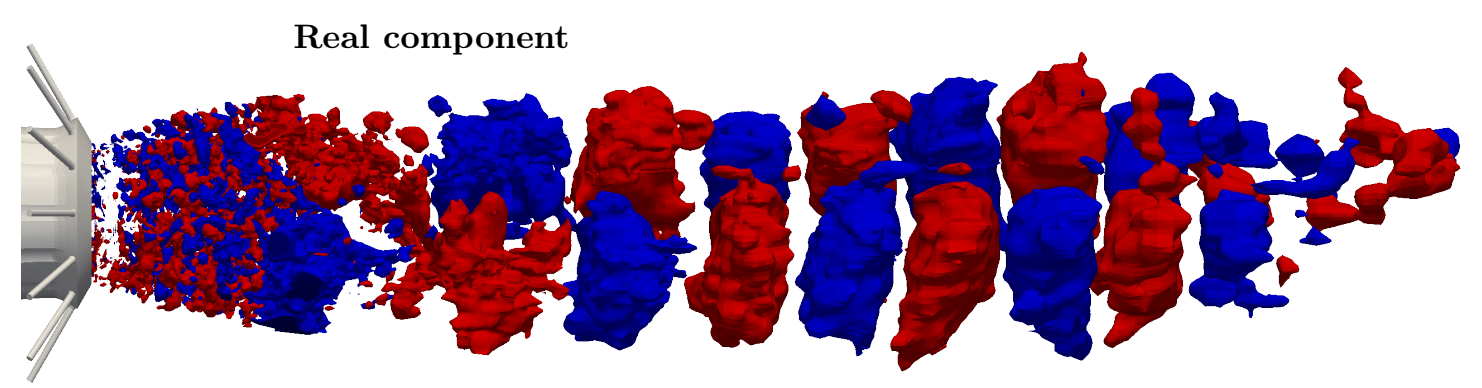

Imaginary component

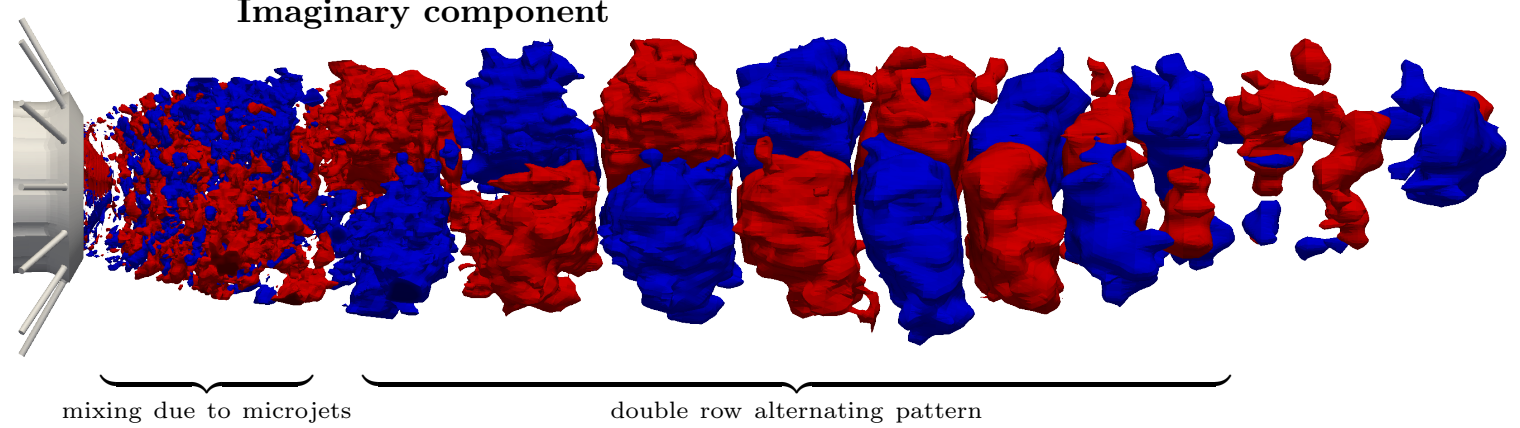

Fig. 13 Positive and negative threshold iso-surfaces of the DFT mode show three-dimensional shape at the screech frequency.

value decomposition of the sequence gives $p_{1}^{N-1}=U \Sigma V^{*}$ (where $V^{*}$ is the conjugate transpose of $V$ ), which leads to,

$$
p_{2}^{N}=\mathcal{D} U \Sigma V^{*} \quad \Rightarrow \quad U^{*} p_{2}^{N} V \Sigma^{-1}=U^{*} \mathcal{D} U
$$

Finally, an eigendecomposition of $U^{*} p_{2}^{N} V \Sigma^{-1}$ can be computed, which approximates the eigenvalues, $\lambda$, and eigenvectors, $\chi$, of $\mathcal{D}$. The frequency of the dynamic mode can be obtained by $2 \pi f_{j}=\log \left(\operatorname{Im}\left(\lambda_{j}\right)\right) / \Delta t$. The dynamic mode shapes, $\mathcal{P}$, can be obtained by the projection of the eigenvector $\chi_{j}$,

$$
\mathcal{P}=U \chi
$$

Although the calculation procedures of the DMD and the DFT modes are different, Chen et al. 31] showed that mean subtracted DMD modes can be equivalent to DFT modes. Comparing the DMD and DFT mode magnitudes and phases in Fig. 12 shows that these exhibit similar characteristics. The highest DMD mode magnitudes can be observed in the shear layer of the $4^{\text {th }}$ and $5^{\text {th }}$ shock cell and the DMD phase angle exhibit the same trends. Moreover, Fig. 14 shows that the three-dimensional DMD mode shape associated with screech reveals a double row alternating pattern of positive and negative amplitudes, similar to the DFT mode shape. The DMD pattern appears to form a helical structure in the nozzle-near shock cells and is helically distorted around the jet axis. 


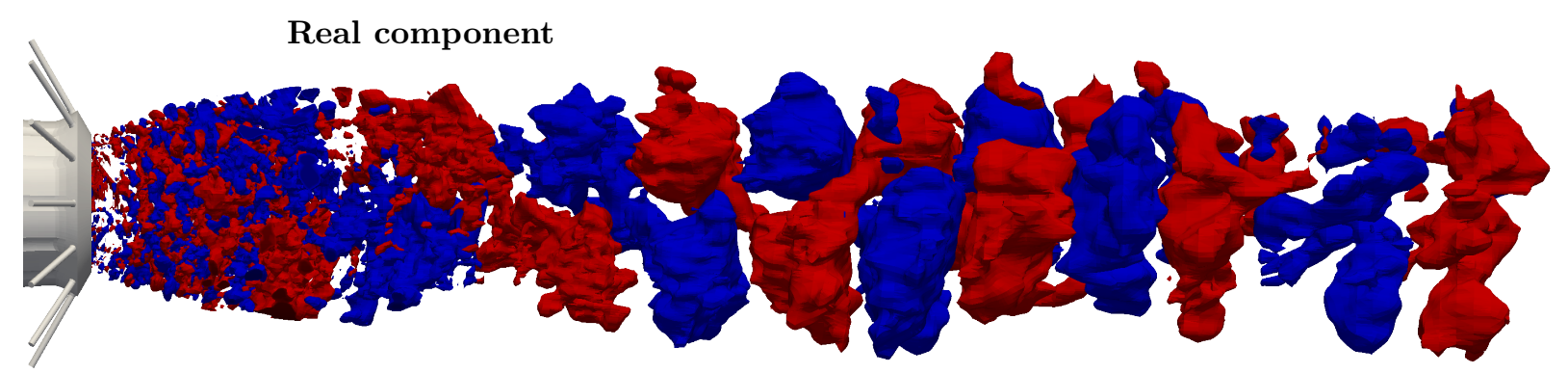

Imaginary component

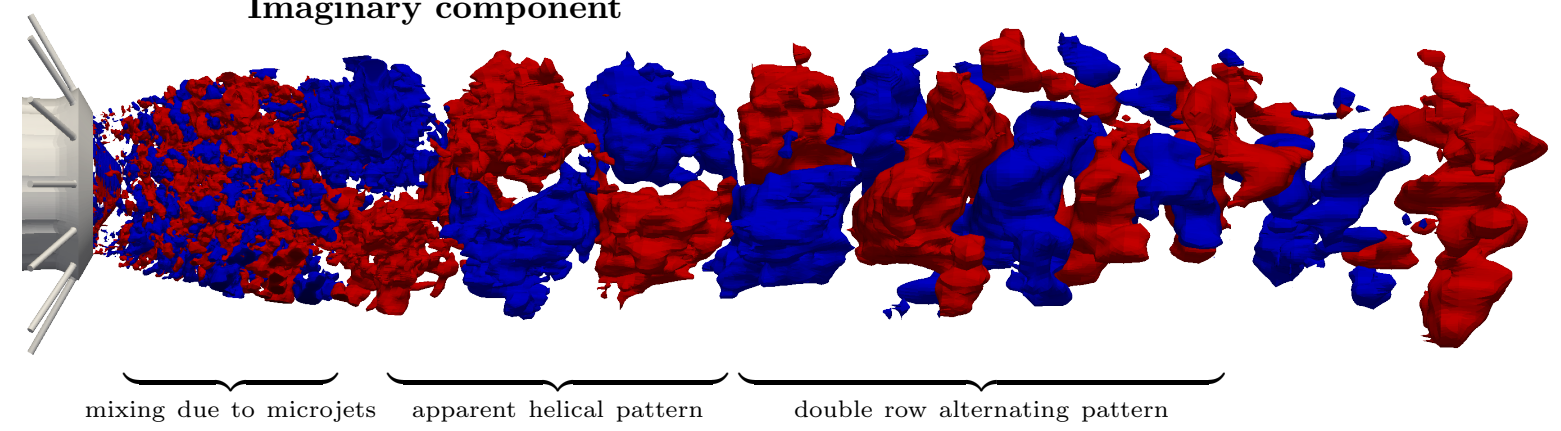

Fig. 14 Illustration of the three-dimensional DMD mode shape associated with screech by showing positive and negative threshold iso-surfaces.

\section{Conclusions}

With the aim to understand the non-linear relationship between screech tone amplitude and fluidic injection pressure, which has been observed in experiments, numerical simulations of the non-ideal expanded supersonic jet have been performed. The non-linear response of the screech tone amplitude suggests that fluidic injection does not only influence the acoustic noise generated by the imposed streamwise vortical structures. It was shown that fluidic injection also translates the shock pattern in the exhaust plume, which was found to be the crucial factor modulating the shock noise component.

With convergent-divergent nozzles, two shock diamond patterns are generated, one originating from the nozzle throat and another from the nozzle exit. It was shown that the two shock pattern can be shifted with respect to each other by varying the fluidic injection pressure. Maximal intersection leads to the lowest pressure fluctuations at the locations of the shocks, and the maximal overlap enhanced the shock noise and the pressure oscillations in the exhaust jet.

The screech tone frequency remained equal, although fluidic injection changed the shock location of the shock pattern originating from the nozzle exit. In contrary, the spacing of the shock pattern originating from the nozzle throat was hardly altered. The intensified averaged pressure fluctuations at the $4^{\text {th }}$ and $5^{\text {th }}$ shock cell were found to be part of the shock pattern originating from the nozzle throat. Therefore, the screech tone frequency is thought to be dominated by the shock pattern originating from the nozzle throat.

Lateral shock cell motion was observed towards the end of the potential core causing intensified averaged 
pressure fluctuations. Discrete Fourier transform and dynamic mode decomposition analysis led to identical conclusions revealing the coherent flow structures causing the lateral distortion of the exhaust jet. The screech mode amplitudes grow radially while convecting in a pulsatile manner in the shear layer downstream. The largest screech mode magnitude occurs at the $4^{\text {th }}$ and $5^{\text {th }}$ shock cell. Before reaching the end of the potential core, the screech mode magnitude decayed and its phase starts reversing direction.

\section{Acknowledgment}

This work was supported by the Swedish National Infrastructure for Computing (SNIC 002-12-11 \& SNIC 2013-11-19) via HPC2N and PDC.

\section{References}

[1] Bridges, J., and Brown, C., "Parametric testing of chevrons on single flow hot jets," 10th AIAA/CEAS Aeroacoustics Conference, 2004, p. 2824. doi:10.2514/6.2004-2824.

[2] Henderson, B., and Bridges, J., "An MDOE investigation of chevrons for supersonic jet noise reduction," $A I A A$ paper, Vol. 3926, 2010, p. 2010. doi:10.2514/6.2010-3926.

[3] Rask, O., Kastner, J., and Gutmark, E., "Understanding How Chevrons Modify Noise in Supersonic Jet with Flight Effects," AIAA journal, Vol. 49, No. 8, 2011, pp. 1569-1576. doi:10.2514/1.J050628.

[4] Bridges, J., Wernet, M., and Brown, C., "Control of jet noise through mixing enhancement," NASA Report No. NASA/TM, Vol. 212335, 2003.

[5] Alkislar, M. B., Krothapalli, A., and Butler, G., "The effect of streamwise vortices on the aeroacoustics of a Mach 0.9 jet," Journal of Fluid Mechanics, Vol. 578, 2007, pp. 139-169. doi:10.1017/S0022112007005022.

[6] Henderson, B., "Fifty years of fluidic injection for jet noise reduction," International Journal of Aeroacoustics, Vol. 9, No. 1, 2010, pp. 91-122. doi:10.1260/1475-472X.9.1-2.91.

[7] Liu, J., Kailasanath, K., Ramamurti, R., Munday, D., and Gutmark, E., "Large-Eddy Simulations of a Supersonic Jet with Fluidic Injection for Noise Reduction," 16th AIAA/CEAS Aeroacoutics Conference, 2010. doi:10.2514/6. 2010-4024.

[8] Morris, P. J., McLaughlin, D. K., and Kuo, C.-W., "Noise reduction in supersonic jets by nozzle fluidic inserts," Journal of Sound and Vibration, Vol. 332, No. 17, 2013, pp. 3992-4003. doi:10.1016/j.jsv.2012.11.023.

[9] Cuppoletti, D. R., and Gutmark, E., "Fluidic Injection on a Supersonic Jet at Various Mach Numbers," $A I A A$ journal, Vol. 52, No. 2, 2014, pp. 293-306. doi:10.2514/1.J010000.

[10] Henderson, B. S., and Norum, T. D., "Impact of azimuthally controlled fluidic chevrons on jet noise," 14th AIAA/CEAS Aeroacoustics Conference, 2008. doi:10.2514/6.2008-3062. 
[11] Cuppoletti, D. R., Malla, B., Gutmark, E. J., Hafsteinsson, H. E., Eriksson, L.-E., and Prisell, E., "The Response of Supersonic Jet Noise Components to Fluidic Injection Parameters," 19th AIAA/CEAS Aeroacoustics Conference, 2013, p. 2196. doi:10.2514/6.2013-2196.

[12] Semlitsch, B., and Mihaescu, M., "Fluidic Injection Scenarios for Shock Pattern Manipulation in Exhausts," AIAA journal, 2018. doi:10.2514/1.J057537.

[13] Suzuki, T., and Lele, S. K., "Shock leakage through an unsteady vortex-laden mixing layer: application to jet screech," Journal of Fluid Mechanics, Vol. 490, 2003, pp. 139-167. doi:10.1017/S0022112003005214.

[14] Edgington-Mitchell, D., Oberleithner, K., Honnery, D. R., and Soria, J., "Coherent structure and sound production in the helical mode of a screeching axisymmetric jet," Journal of Fluid Mechanics, Vol. 748, 2014, pp. 822-847. doi:10.1017/jfm.2014.173.

[15] Harper-Bourne, M., and Fisher, M., "The noise from shock waves in supersonic jets," AGARD-CP-131, Vol. 11, 1974, pp. 1-13.

[16] Tam, C., and Tanna, H., "Shock associated noise of supersonic jets from convergent-divergent nozzles," Journal of Sound and Vibration, Vol. 81, No. 3, 1982, pp. 337-358. doi:10.1016/0022-460X(82)90244-9.

[17] Tam, C., Seiner, J., and Yu, J., "Proposed relationship between broadband shock associated noise and screech tones," Journal of Sound and Vibration, Vol. 110, No. 2, 1986, pp. 309-321. doi:10.1016/S0022-460X(86)80212-7.

[18] Berland, J., Bogey, C., and Bailly, C., "Numerical study of screech generation in a planar supersonic jet," Physics of Fluids, Vol. 19, No. 7, 2007, p. 075105. doi:10.1063/1.2747225.

[19] Panda, J., "Shock oscillation in underexpanded screeching jets," Journal of Fluid Mechanics, Vol. 363, 1998, pp. $173-198$

[20] Suda, H., Manning, T. A., and Kaji, S., "Transition of oscillation modes of rectangular supersonic jet in screech," AIAA, Aeroacoustics Conference, 15 th, Long Beach, CA, 1993. doi:10.2514/6.1993-4323.

[21] Gojon, R., Bogey, C., and Mihaescu, M., "Oscillation Modes in Screeching Jets," AIAA Journal, Vol. 56, No. 7, 2018, pp. 2918-2924. doi:10.2514/1.J056936.

[22] Powell, A., "On the mechanism of choked jet noise," Proceedings of the Physical Society. Section B, Vol. 66, No. 12, 1953, p. 1039. doi:10.1088/0370-1301/66/12/306.

[23] Westley, R., and Woolley, J., "The near field sound pressures of a choked jet when oscillating in the spinning mode," AIAA paper, Vol. 479, 1975, p. 1975. doi:10.2514/6.1975-479.

[24] Callender, B., Gutmark, E., and Dimicco, R., "The design and validation of a coaxial nozzle acoustic test facility," AIAA paper, Vol. 369, 2002. doi:10.2514/6.2002-369. 
[25] Jameson, A., Schmidt, W., Turkel, E., et al., "Numerical solutions of the Euler equations by finite volume methods using Runge-Kutta time-stepping schemes," AIAA paper, Vol. 1259, 1981, p. 1981. doi:10.2514/6.1981-1259.

[26] Uzun, A., Blaisdell, G. A., and Lyrintzis, A. S., "Sensitivity to the Smagorinsky constant in turbulent jet simulations," AIAA journal, Vol. 41, No. 10, 2003, pp. 2077-2079. doi:10.2514/2.1900.

[27] Semlitsch, B., and Mihaescu, M., "Consequence of sub-grid scale modeling onto the prediction of acoustic noise emission," 6th European Conference on Computational Fluid Dynamics (ECFD VI), 2014.

[28] Margolin, L. G., and Rider, W. J., "A rationale for implicit turbulence modelling," International Journal for Numerical Methods in Fluids, Vol. 39, No. 9, 2002, pp. 821-841. doi:10.1002/fld.331.

[29] Semlitsch, B., Mihaescu, M., Fuchs, L., and Gutmark, E., "Large Eddy Simulations of Microjets Impact on Supersonic Jet Exiting a CD Conical Nozzle," 19th AIAA/CEAS Aeroacoustics Conference (34th AIAA Aeroacoustics Conference), 2013, p. 17. doi:10.2514/6.2013-2139.

[30] Mercier, B., Castelain, T., and Bailly, C., "Experimental characterisation of the screech feedback loop in underexpanded round jets," Journal of Fluid Mechanics, Vol. 824, 2017, pp. 202-229. doi:10.1017/jfm.2017.336.

[31] Chen, K. K., Tu, J. H., and Rowley, C. W., "Variants of dynamic mode decomposition: boundary condition, Koopman, and Fourier analyses," Journal of nonlinear science, Vol. 22, No. 6, 2012, pp. 887-915. doi:10.1007/ s00332-012-9130-9. 